\title{
Escherichia coli produtora de toxina shiga (STEC): principais fatores de virulência e dados epidemiológicos
}

\author{
Shiga toxin-producing Escherichia coli (STEC): \\ principal virulence factors and epidemiology
}

\author{
Ariane Mayumi Saito Bertão ${ }^{1}$, Halha Ostrensky Saridakis ${ }^{2}$
}

\begin{abstract}
Resumo
Escherichia coli produtora de toxina Shiga (STEC) é um importante patógeno veiculado por alimentos, principalmente produtos derivados de carne bovina e está associado a quadros de diarréias leves a severas e sanguinolentas. Em alguns indivíduos, a infecção por STEC pode progredir para a síndrome hemolítico-urêmica (HUS), seqüela caracterizada pela falência renal e a púrpura trombocitopênica trombótica (TTP), com possível envolvimento do sistema nervoso central. O gado bovino, geralmente saudável, é o principal reservatório de STEC, embora estas cepas também tenham sido isoladas de outros animais domésticos: ovelhas, cabras, cães, gatos e suínos. A principal característica de virulência, a produção de toxinas Shiga, não é suficiente para causar doenças e outros fatores são considerados relevantes, como a produção de enterohemolisina e de adesinas fimbriais e afimbriais. Embora as doenças humanas associadas a STEC sejam pouco descritas no Brasil, podemos observar uma significativa ocorrência destas cepas nos rebanhos bovinos, bem como a correlação entre sorotipos encontrados nestes animais e em pacientes humanos.

Palavras-chave: Escherichia coli produtora de toxina Shiga (STEC). Diarréia. Bovinos. Fatores de virulência.
\end{abstract}

\begin{abstract}
Shiga toxin producing Escherichia coli is an important food borne pathogen, mainly beef products, and is associated to mild and severe bloody diarrhea. In some individuals, STEC infection can progress to hemolytic-uremic syndrome (HUS), a sequela characterized by renal failure, and thrombotic thrombocytopenic purpura (TTP), with possible central nervous system involvement. Cattle, usually healthy, is the principal reservoir of STEC, although these strains have also been isolated from other domestic animals: sheep, goats, dogs, cats and pigs. The principal virulence feature, the production of Shiga toxins, is not enough to cause diseases, and other factors are considered important, as enterohemolysin and fimbrial and afimbrial adhesions production. Although human diseases associated to STEC have not been frequently reported in Brazil, their presence is frequent in cattle, as well as the correlation between serotypes found in these animals and human patients.
\end{abstract}

Key words: Shiga toxin producing Escherichia coli (STEC). Diarrhea. Cattle. Virulence factors.

\footnotetext{
1 Farmacêutica bioquímica, graduada e mestre em Microbiologia pela Universidade Estadual de Londrina. E-mail: arianesaito@yahoo.com.br

2 Departamento de Microbiologia, Universidade Estadual de Londrina, Londrina-PR, Brasil.
} 


\section{Introdução}

Escherichia coli foi descrita em 1885 como uma bactéria componente da microbiota intestinal por $\mathrm{T}$. Escherich, que a denominou Bacterium coli commune (ROBINS-BROWNE, 1987). Somente em 1945, entretanto, essa bactéria foi definitivamente associada à diarréia infantil por Bray (1945), e por ele denominada Bacterium coli neapolitanum. Esse fato também comprovado por outros pesquisadores (GILES; SANGSTER, 1948; SMITH, 1949).

E. coli é classificada sorologicamente em sorogrupos e sorotipos com base na sua composição antigênica: antígenos $\mathrm{O}$ ou somáticos para os sorogrupos, e antígenos flagelares ou $\mathrm{H}$ para os sorotipos. Ela pode, ainda, expressar antígenos $\mathrm{K}$ ou capsulares, importantes na patogênese (CAMPOS et al., 2004). Este esquema, O:H:K, estabelecido por Kauffmann em1947, possibilitou um grande avanço na identificação de $E$. coli e, na aplicação deste sistema, verificou-se que Bacterium coli neapolitanum pertencia ao sorogrupo O111.

Embora E. coli seja a bactéria anaeróbia facultativa predominante na mucosa intestinal dos mamíferos saudáveis, coexistindo sem causar danos ao hospedeiro, várias cepas, ao adquirir fatores de virulência específicos, tornaram-se potencialmente danosas (NATARO; KAPER, 1998). E. coli produtora de toxina Shiga (STEC), apresenta seus principais fatores de virulência codificados por genes localizados em elementos genéticos móveis. Ela está relacionada a um amplo espectro de doenças humanas, que compreende desde diarréias leves à colite hemorrágica (HC) e a síndrome hemolíticourêmica (HUS, cuja possível seqüela mais grave é, possivelmente, a falência renal) e a púrpura trombocitopênica trombótica (TTP), em seres humanos (NATARO; KAPER, 1998; GRIFFIN; TAUXE, 1991). Humanos podem ser infectados por STEC ao ingerir alimentos de origem animal contaminados, principalmente a carne bovina, o que tornou esta categoria de E. coli objeto de estudos na medicina humana, veterinária e na biologia molecular (MAINIL; DAUBE, 2005).
A proposta desta revisão é apresentar a história e as definições de STEC, descrevendo os fatores de virulência destas cepas e dados epidemiológicos do Brasil e de outros países.

\section{História e Definições}

O reconhecimento de STEC como uma classe de E. coli patogênica distinta foi o resultado de duas linhas de estudo convergentes: a primeira iniciou-se quando a capacidade de certas cepas de E. coli, pertencentes aos sorogrupos $\mathrm{O} 18, \mathrm{O} 26, \mathrm{O} 111, \mathrm{O} 126$ e $\mathrm{O} 128$, de produzir toxinas que causavam efeito citopático irreversível em células Vero (células de rim de macaco verde africano), foi descrita pela primeira vez por Konowalchuk, Speirs e Starvic em 1977, surgindo o termo verotoxina ou verocitotoxina. Neste trabalho, das 10 cepas citotóxicas, 3 foram isoladas de leitões acometidos com a doença do edema e 7 de crianças com diarréia, sugerindo o envolvimento desta nova citotoxina na patogênese de doenças gastrintestinais (KONOWALCHUK; SPEIRS; STARVIC, 1977). Em 1982, O’Brien et al. descreveram a capacidade de cepas de EPEC em produzir uma citotoxina letal para células HeLa. Assim como a toxina de Shiga, esta inibia a síntese protéica em células HeLa, era enterotóxica para intestino de coelho e letal para camundongos. Por apresentar essas características, foi chamada toxina Shiga-like. As nomenclaturas "VTEC/verotoxina" passaram a ser utilizadas, principalmente, por pesquisadores canadenses, enquanto os termos "SLTEC/ SLT" foram adotados por pesquisadores americanos. Em 1996, Calderwood et al propuseram a nomenclatura toxina Shiga/E. coli produtora de toxina Shiga (Stx/STEC), devido ao alto grau de homologia estutural e funcional da toxina de E. coli com a toxina de Shigella dysenteriae tipo 1.

A segunda via teve início em 1983, com o relato de que um raro sorotipo de E. coli, O157:H7, seria responsável por um surto de diarréia sanguinolenta, ligado ao consumo de hambúrgueres contaminados e mal-cozidos. Este quadro clínico foi denominado 
colite hemorrágica (HC) (RILEY et al., 1983). Subseqüentemente, O’Brien e La Veck (1983) purificaram SLT e demonstraram que a toxina Shiga produzida por $S$. dysenteriae tipo 1 e SLT (E. coli cepa H30, da coleção de Konowalchuck) eram idênticas, tanto em suas características bioquímicas como atividades biológicas. Em outro trabalho, O'Brien et al. (1983) verificaram que a cepa de $E$. coli sorotipo O157:H7 descrita por Riley (1983) também a produzia. Nos anos seguintes, E. coli O157:H7 foi associada a quadros de diarréia leves a severas e sanguinolentas, com seqüelas em alguns pacientes como a síndrome hemolítico-urêmica (HUS), que pode levar à falência renal (KARMALI et al., 1983) e a púrpura trombocitopênica trombótica (TTP), com possível envolvimento do sistema nervoso central. Devido à associação inicial de O157:H7 com colite hemorrágica, as cepas pertencentes a este sorotipo foram denominadas Escherichia coli enterohemorrágicas (EHEC) e, por muitos anos, EHEC permaneceu como sinonímia de E. coli O157:H7, agente etiológico de HC. Atualmente, o termo STEC é utilizado para caracterizar cepas de E. coli produtoras de Stx, enquanto EHEC caracteriza cepas de E. coli que produzem Stx e causam uma lesão histopatológica (attaching and effacing, A/E) (MAINIL; DAUBE, 2005). As cepas de STEC também são denominadas EHEC LEEnegativas ou eae-negativas (LUCK et al., 2005).

\section{Fatores de virulência}

Os mecanismos pelos quais STEC causam HC e HUS não foram totalmente elucidados. A mais importante característica de virulência, a produção de uma ou mais toxinas Shiga, isoladamente, não é suficiente para causar doenças e outros fatores são considerados relevantes, como a presença do plasmídio pO157, que codifica a enterohemolisina e a produção de adesinas fimbriais e afimbriais (NATARO; KAPER, 1998; PATON; PATON, 1998).

\section{Toxinas Shiga}

Ensaios imunológicos de neutralização demonstraram que as toxinas Shiga, principais marcadores de virulência de cepas de STEC, podem ser divididas em dois grupos antigênicos. $\mathrm{O}$ grupo um, Stx1, é neutralizado por anti-soro da citotoxina de $S$. dysenteriae 1, pois são consideradas idênticas (O'BRIEN; LA VECK, 1983) O grupo 2, Stx2, é neutralizável apenas por anti-Stx2 (O’BRIEN; HOLMES, 1987). Os genes estruturais para Stx1 e Stx2 são encontrados em fagos lambda temperados, porém os genes para Stx2 já foram encontrados em cromossomos e apresentam 55 e $57 \%$ das sequiências genéticas de suas subunidades A e B idênticas, respectivamente (JACKSON et al., 1987). Stx1 é considerada altamente conservada e suas variantes apresentam poucas diferenças nas suas seqüências genéticas, sem maiores conseqüências nas suas propriedades antigênicas e citotóxicas (MELTONCELSA; O'BRIEN, 1998; DUFFY et al., 2001; THORPE et al., 2002). No entanto, algumas variantes de Stx1 foram descritas: Stx1c e Stx1OX3 encontradas em amostras de E. coli isoladas de humanos e ovinos (KOCH et al., 2001; ZHANG et al., 2002) e Stx1d, de amostras bovinas (BÜRK et al., 2003). Cinco variantes biológicas de Stx 2 foram descritas, as quais diferem umas das outras por sua antigenicidade, toxicidade e seqüência genética (MELTON-CELSA; O'BRIEN, 1998; DUFFY et al., 2001; THORPE et al., 2002): Stx2, Stx2c, Stx2d e Stx2f (anteriormente denominada Stx2ev) isoladas de humanos e Stx2e, isolada de suínos.

Stx1 e Stx2 apresentam outras diferenças entre si, como a afinidade pelo receptor em comum, Gb3 e os mecanismos de regulação da expressão: Stx1 possui uma afinidade cerca de 10 vezes maior por Gb3, se comparado a Stx2 (HEAD et al., 1991). No entanto, estudos mais recentes, utilizando métodos automatizados, revelaram uma velocidade de dissociação menor entre Gb3 e Stx2 do que Stx1. Assim, tanto a ligação quanto a dissociação entre o receptor e Stx2 parece ocorrer de forma mais lenta. 
Estas características podem explicar a maior associação entre Stx2 e danos vasculares, quando este é comparado a Stx1 (NAKAJIMA et al., 2001).

A estrutura A-B da família das toxinas Shiga, holotoxinas de aproximadamente 70kDa,é conservada entre seus membros. A subunidade A, de $32 \mathrm{kDa}$, pode ser clivada proteoliticamente, resultando em dois peptídios: A1 e A2, de 28 e 4kDa, respectivamente, e que permanecem ligados por uma ligação dissulfeto. $\mathrm{O}$ peptídio A1 apresenta atividade enzimática $(\mathrm{N}$ glicosidase), enquanto $\mathrm{A} 2$ tem a função de ligar a subunidade A ao pentâmero formado por cinco subunidades $\mathrm{B}$ idênticas, de $7,7 \mathrm{kDa}$ cada. O pentâmero B liga a toxina ao receptor glicolipídico específico, presente na superfície da célula eucariótica, globotriasilceramida (Gb3) para Stx1 e Stx 2 e globotetrasilceramida (Gb4) para Stx2e. Após a ligação, a holotoxina sofre endocitose por meio de depressões da membrana citoplasmática da célula eucariótica revestidas pela proteína clatrina (coated pits), formando uma vesícula selada com a holotoxina em seu interior. No citoplasma, esta vesícula pode se fundir com lisossomos, resultando na degradação da toxina. Porém, em células eucarióticas sensíveis a Stx, as vesículas são transportadas até o retículo endoplasmático via complexo de Golgi, alcançando, então, o citosol. Durante este processo, a subunidade A é clivada pela enzima furina, gerando o fragmento cataliticamente ativo, A1 e o fragmento A2, cuja ligação dissulfeto é posteriormente reduzida. $\mathrm{O}$ fragmento A1 livre tem atividade RNA N-glicosidase, e interage com a unidade $60 \mathrm{~S}$ do ribossomo. Ao remover um resíduo de adenina da subunidade $28 \mathrm{~S}$ do rRNA eucariótico, A1 livre impede a ligação do amino-acil-RNA transportador a esta subunidade, e inibe a etapa de elongamento da síntese protéica e, assim, causa a morte celular (PATON; PATON, 1998). A toxina Shiga, assim como a toxina colérica, não é capaz de formar poros e alcançar o citosol da célula-alvo.

As Stxs são produzidas no cólon e, através da corrente sangüínea, são levadas aos rins, causando danos ao endotélio vascular e oclusão dos microvasos, através de uma combinação de toxicidade direta e indução local da inflamação local os quais podem levar à HUS (ANDREOLI et al, 2002).

Habib e Jackson (1993) descreveram os operons de Stxs: uma estrutura em comum, que consiste em uma única unidade transcricional, codificando primeiro a subunidade A, seguida da subunidade B. A tradução da subunidade B ocorre de forma mais intensa do que a subunidade $\mathrm{A}$, devido, aparentemente, à maior afinidade do ribossomo ao seu local de ligação, rbs (ribossome binding site), satisfazendo a relação estequiométrica A1:B5 da holotoxina.

\section{Enterohemolisina (EHEC-Ehly)}

Em 1989, Beutin et al., estudando cepas de STEC, observaram que uma cerca de $89 \%$ apresentavam um fenótipo hemolítico diferente do padrão já conhecido e associado à alfa-hemolisina de E. coli (Hly). As cepas foram denominadas enterohemolíticas e não produziam alfa-hemólise em ágar sangue de carneiro convencional, mas pequenas e turvas zonas hemolíticas, após a incubação por 18 a 24 horas em ágar sangue com hemácias de carneiro lavadas, suplementado com cloreto de cálcio. Enquanto a alfa-hemolisina é codificada por genes cromossomais, os genes que codificam a enterohemolisina foram descritos em um plasmídio de $60 \mathrm{MDa}$, pO157, na cepa EDL 933 (SCHMIDT; KARCH; BEUTIN, 1994). Embora a enterohemolisina esteja presente na maioria dos isolados de EHEC/STEC e a enterohemólise sugerida como um marcador para detecção de EHEC (BEUTIN et al., 1996), sua contribuição na patogenicidade de EHEC/STEC ainda não foi esclarecida. Sugeriu-se que a lise de eritrócitos pode fornecer ferro e estimular o crescimento de STEC (LAW; KELLY, 1995). O plasmídio pO157 está presente também em cepas do sorotipo O26:H11 e na maioria de STEC isoladas de humanos (BEUTIN et al., 1994; LEVINE et al., 1987), seus genes codificam outros possíveis marcadores de virulência adicionais: uma catalase-peroxidase (KatP) 
(BRUNDER et al., 1996) e uma serina protease (EspP), capaz de clivar o fator $\mathrm{V}$ de coagulação humano e de exacerbar a doença hemorrágica (BRUNDER et al., 1997).

\section{Fatores de adesão}

\section{Intimina}

Em EHEC, assim como em EPEC, outros fatores de virulência estão localizados cromossomalmente em uma ilha de patogenicidade denominada locus of enterocyte effacement (LEE), cujos genes codificam os elementos responsáveis pela lesão intestinal A/E (attaching and effacing). Essa lesão é caracterizada pela degeneração localizada das microvilosidades epiteliais intestinais e a montagem de estruturas semelhantes a pedestais, constituídas de filamentos de actina, formadas nos locais onde há bactérias aderidas (NATARO; KAPER, 1998). Os genes da região LEE codificam uma adesina não fimbrial de $94 \mathrm{kDa}$, a intimina (eae); um sistema de secreção do tipo III (proteínas Esc e Sep); chaperoninas (proteínas Ces); proteínas translocadoras (EspA, EspB e EspD) e proteínas efetoras (EspF, EspG e Map), assim como o receptor para intimina (Tir). A função de muitos quadros abertos de leitura (open reading frames - ORFs) ainda é desconhecida. (GIRARD et al, 2005). A intimina é fator-chave de colonização para EHEC em ovinos (CORNICK et al., 2002) e bovinos recémnascidos e adultos (DEAN-NYSTROM et al., 1998), e parece ser a única adesina de E. coli O157:H7 cujo papel na colonização do epitélio intestinal foi demonstrado em modelos animais (DONNENBERG et al., 1993, McKEE et al., 1995). No entanto, mutantes intimina-negativos ainda são capazes de colonizar certos locais do trato gastrintestinal dos ruminantes, e isso indica que outros fatores de colonização podem estar presentes (VAN DIEMEN et al., 2005). Em humanos, STEC eae positivos estão relacionados a quadros severos de diarréia, principalmente HC, e HUS (KARMALI, 1989; PATON; PATON, 1998). A região 5' do gene eae é conservada, enquanto a região 3' é heterogênea. Esta observação tornou possível a confecção de oligonucleotídios iniciadores universais e aleloespecíficos para o ensaio de PCR (polymerase chain reaction), permitindo, assim, a diferenciação de 15 variantes de eae, que codificam 15 diferentes tipos e subtipos de intimina: $\alpha 1, \alpha 2, \beta 1, \beta 2, \gamma 1, \gamma 2 / \theta, \delta /$ $\kappa, \varepsilon, \zeta, \eta, \imath, \lambda, \mu, \nu$, e $\xi$ (BLANCO et al., 2004).

\section{Adesinas fimbriais de EHEC}

A análise da sequiência genômica de duas cepas protótipos do sorotipo O157:H7 indicou a existência de, no mínimo, 12 regiões que codificam sequiências homólogas a adesinas (HAYASHI et al., 2001; PERNA et al., 2001). Uma dessas regiões contém genes proximamente relacionados ao operon lpf (fimbria longa polar, LP) de Salmonella enterica serovar Typhimurium (PERNA et al., 2001). A introdução do operon EHEC lpf em E. coli K-12 resultou em um aumento da expressão de fímbrias e da adesão a células em cultivo (TORRES et al., 2002a). A subunidade principal de LP, lpfA, foi detectada em cepas de EHEC dos sorogrupos O157 e 0145 (SZALO et al., 2002).

\section{Adesinas não-fimbriais de EHEC}

Neste grupo estão incluídas as adesinas Efa1 (EHEC factor for adherence) (NICHOLLS et al., 2000; STEVENS et al., 2002), Iha (adesina homóloga a IrgA, de Vibrio cholerae) (TARR et al., 2000), Cah (homólogo ao antígeno 43 ligante de cálcio) (TORRES et al., 2002b) e OmpA (proteína de membrana externa A) (TORRES; KAPER, 2003). Estas proteínas expressaram suas propriedades de adesão somente em experimentos recombinantes e seu papel na colonização do trato gastrintestinal requer maiores estudos.

\section{Adesinas fimbriais e não-fimbriais de STEC}

As cepas de STEC não possuem a região LEE, portanto a ausência de intimina indica que outras 
adesinas devem estar envolvidas na adesão às células hospedeiras.

A adesina Saa (adesina autoaglutinante de STEC) foi descrita em uma cepa de STEC do sorotipo O113:H21. Saa é uma proteína de membrana externa, associada ao aumento da adesão em células HEp-2 quando expressa como uma proteína recombinante em E. coli (PATON et al., 2001). Estudos recentes indicaram que cepas saa-positivas são mais freqüentemente encontradas em STEC isolados de bovinos do que de humanos, sugerindo um papel importante desta adesina na colonização intestinal desses animais (JENKINS et al., 2003).

A fímbria LP também foi caracterizada em cepas STEC O113:H21 e o gene $l p f A_{O 13}$ foi detectado em diversas cepas de E. coli isoladas de humanos e animais (OSEK et al., 2003).

\section{Dados epidemiológicos}

HUS é considerada uma doença de origem alimentar e foi inicialmente relacionada ao consumo de hambúrgueres, carne moída e laticínios contaminados com STEC (RILEY, 1987). Nos Estados Unidos, a categoria STEC está associada a, aproximadamente, 110.000 casos de doenças e 90 mortes, anualmente (MEAD et al., 1999). No Brasil, de acordo com os registros da AIH/DATASUS/MS, foram notificados 12 casos de HUS no estado de São Paulo no período de 1998/2000, com história anterior de diarréia e possível, porém não confirmado, envolvimento com E. coli O157:H7 (BRASIL, 2001). O primeiro relato de isolamento de STEC de um paciente com HUS ocorreu em um hospital da cidade de São Paulo, no ano de 2001 (GUTH, et al., 2002): uma criança, com história de diarréia aguda ocorrida três semanas antes da internação, cujos sintomas clínicos e resultados de exames laboratoriais levaram ao diagnóstico desta síndrome. A cepa isolada pertencia ao sorotipo O26:H11. Vaz et al. (2004), analisando uma coleção de 39 cepas de STEC isoladas de pacientes humanos com diarréia no período de 1976 a 1999, em São Paulo, Brasil, detectou a predominância dos sorogrupos O111 e O26. Os sorotipos encontrados foram: O26:H11, O55:H19, O93:H19, O111:NM, O11:H11, O118:H16 e O157:H7.

Embora o sorotipo 0157:H7 esteja ligado à maioria dos surtos associados a STEC descritos mundialmente, estudos recentes indicam que a incidência deste sorotipo vem decaindo substancialmente (CDC, 2004). Em contraste, STEC não-O157 vem sendo isolada com maior frequiência à partir de amostras fecais de pacientes com HUS (BANATVALA et al., 2001; JAEGER et al., 2000), entre os quais os sorogrupos O26, O91, O103, O111 e O113 (NATARO; KAPER, 1998; PATON; PATON, 1998) No Brasil, poucas cepas O157:H7 foram isoladas de humanos, no estado de São Paulo. Esses pacientes não apresentavam diarréia sanguinolenta (IRINO et al, 2002).

$\mathrm{O}$ primeiro surto de colite hemorrágica ligado a EHEC sorotipo O157:H7 estava relacionado ao consumo de hambúrgueres contaminados, levando à suspeita de um reservatório bovino (RILEY et al., 1983). Desde então, estudos em diferentes países têm demonstrado que o gado bovino, geralmente saudável, é o principal reservatório de STEC/EHEC: na Argentina, segundo estudos de Sanz et al. (1998), a taxa de isolamento de STEC de bovinos adultos tratados a pasto foi de $22 \%$ no campo e, no abatedouro, 44\%. Já Padola et al. (2004) isolaram STEC e EHEC sorotipo O157:H7em 63 e 6,8\%, respectivamente, dos animais de uma fazenda de confinamento. Na Espanha, STEC foi isolada em $37 \%$ dos bezerros e em $35 \%$ dos bovinos adultos investigados (BLANCO et al., 1997). Nos Estados Unidos, a prevalência de EHEC sorotipo O157:H7 no gado saudável varia de entre 2 a $20 \%$ dos animais (MAINIL; DAUBE, 2005). Esses animais podem apresentar um quadro inicial de diarréia quando jovens e, quando se recuperam, tornam-se animais saudáveis, portadores de STEC (VERWEYEN et al., 2000). STEC também foi isolado a partir das fezes de animais domésticos: ovelhas, cabras, cães, gatos e suínos (BEUTIN et al., 1993) 
No Brasil, Cerqueira et al. (1999) analisaram o gado leiteiro e de corte no Estado do Rio de Janeiro e verificaram uma alta ocorrência dos genes stx nestes animais, detectados por PCR: $82 \%$ do gado leiteiro e $53 \%$ do gado de corte, sendo $1,5 \%$ dos isolados EHEC sorotipo O157:H7. Este foi o primeiro relato de EHEC O157:H7 isolado de gado leiteiro no Brasil. Leomil et al. (2003), detectaram, por PCR, a ocorrência dos genes stx em $20 \%$ dos bezerros diarréicos e em 7,8\% dos animais não-diarréicos das fazendas de corte do Estado de São Paulo estudadas. Moreira et al. (2003), analisando fazendas de leite da região de Pelotas, no Estado do Rio Grande do Sul, detectaram STEC em $49 \%$ dos animais por meio do ensaio de citotoxicidade em células Vero. Salvadori et al. (2003) pesquisando bezerros diarréicos da Região Centro-Oeste detectou, por PCR, genes stx em $16.1 \%$ dos animais. Mais recentemente, Irino et al. (2005), isolaram STEC em 25,5\% dos animais pesquisados em fazendas de leite no estado de São Paulo. Os genes stx foram pesquisados por ensaios de hibridização de DNA em colônias. Dois novos sorotipos de STEC foram encontrados: O79:H14 e O98:H17, mas o sorotipo O178:H19, anteriormente descrito, fora recentemente detectado somente na Espanha (BLANCO et al., 2004). Os sorotipos O111:NM e O118:H16 descritos nos estudos de Leomil et al. (2003) e Salvadori et al. (2003), além do sorotipo O111:H8 (LEOMIL, et al., 2003), também foram encontrados em pacientes brasileiros com diarréia (GUTH et al., 2002; VAZ et al., 2004).

Elder et al (2000), examinando gado de corte em frigoríficos nos EUA, antes e após o abate, puderam isolar E. coli $\mathrm{O} 157: \mathrm{H} 7 \mathrm{em} 28 \%$ das fezes, 11 na pele/ couro; e , na carcaça, em $43 \%$ antes da evisceração, $18 \%$ pós-evisceração e $2 \%$ após a lavagem da carcaça, pronta para o resfriamento. Cerqueira et al. (1997) encontraram, no Rio de Jameiro, uma alta prevalência de STEC em produtos cárneos de origem bovina, cerca de $71 \%$.
Embora alimentos de origem bovina sejam considerados os principais veículos da infecção por STEC/EHEC, outros alimentos sujeitos à contaminação por fezes bovinas no cultivo e/ou produção também são potencialmente perigosos, como frutas e vegetais crus, maionese, suco de maçã e salame (PATON; PATON, 1998). O maior surto de EHEC relatado até 1997 ocorreu na cidade de Sakai, Japão, e foi devido ao consumo de brotos de rabanete crus contaminados. Deles resultaram mais de 6000 casos de HC e mais de 100 casos de HUS (SWINBANKS, 1996).

\section{Conclusão}

Embora as graves doenças humanas associadas a STEC tenham sido pouco descritas no Brasil, podemos observar uma significativa ocorrência destas cepas nos rebanhos bovinos, bem como a correlação entre sorotipos encontrados nestes animais e em pacientes humanos. Temos, ainda, a proximidade de nosso Estado com a Argentina, país onde HUS é endêmica. A adoção de medidas de prevenção da contaminação por STEC dos produtos destinados ao consumo humano é, portanto, necessária.

\section{Referências}

ANDREOLI, S. P.; TRACHTMAN, H.; ACHESON, D. W.; SIEGLER, R. L.; OGRIG, T. G. Hemolytic uremic syndrome: epidemiology, pathophysiology and therapy. Pediatric Nephrology, Berlin, v.17, n. 4, p. 293-298, Apr. 2002.

BANATVALA, N.; GRIFFIN, P. M.; BARRETT. T. J.; GREENE, K. D.; BIBB, W. F.; GREEN, J. H.; WELLS, J. G. The United States National prospective hemolytic uremic syndrome study: microbiologic, serologic, clinical and epidemiologic findings. Journal of Infectious Diseases, Chicago, v. 183, n. 7, p. 1063-1070, Mar. 2001.

BEUTIN, L.; MONTENEGRO, M. A.; ORSKOV, I.; ORSKOV, F.; PRADA, J., ZIMMERMANN, S.; STEPHAN, R. Close association of verotoxin (Shiga-like toxin) with enterohemolysin production in strains of Escherichia coli. Journal of Clinical Microbiology, Washington, v.2 , n. 7, p. 2559-2564, Nov. 1989. 
BEUTIN, L.; GEIER, D.; STEINHUCK, H.; ZIMMERMANN, S.; SCHEUTZ, F. Prevalence and some properties of Verotoxin (Shiga-like toxin)-producing Escherichia coli in seven different species of healthy domestic animals. Journal of Clinical Microbiology, Washington, v. 31, n. 9, p. 2480-2488, Sept. 1993.

BEUTIN, L.; ALEKSIC, S.; ZIMMERMANN. S.; GLEIER, $K$. Virulence factors and phenotype traits of verotoxigenic strains of Escherichia coli isolated from humans patients in Germany. Medical Microbiology and Immunology, Berlin, v. 183, n. 1, p. 13-21, Feb. 1994.

BEUTIN, L.; ZIMMERMANN, S.; GLEIER, K. Rapid detection and isolation of Shiga-like toxin (verocytotoxin)producing Escherichia coli by direct testing of individual enterohemolytic colonies from washed sheep blood agar plates in the VTEC-RPLA assay. Journal of Clinical Microbiology, Washington, v. 34, n. 11, p. 2812-2814, Nov. 1996.

BLANCO, M.; BLANCO, J. E.; BLANCO, J.; MORA, A.; PRADO, C.; ALONSO, M. P.; MOURIÑO, M.; MADRID, C.; BALSALOBRE, C.; JUÁREZ, A. Distribution and characterization of faecal verotoxin-producing Escherichia coli (VTEC) isolated from healthy cattle. Veterinary Microbiology, Amsterdam, v. 54, n. 3-4, p. 309319, Mar 1997.

BLANCO, M.; BLANCO, J. E.; MORA, A.; DAHBI, G.; ALONSO, M. P.; GONZÁLEZ, E. A.; BERNÁRDEZ, M. I.; BLANCO, J. Serotypes, virulence genes and intimin types Shiga-toxin (verotoxin)-producing Escherichia coli isolates from cattle in Spain: Identification of a new intimin variant gene (eae- e). Journal of Clinical Microbiology, Washington, v. 42, n. 2, p. 645-651, Feb. 2004.

BRASIL. Centro de Vigilância Epidemiológica/CVE/SESSP. Manual das doenças transmitidas por alimentos, 2001. Disponível em: <http://www.cve.saude.sp.gov.br/htm/ ecolinet.htm>. Acesso em: 20/05/2005.

BRAY, J. Isolation of antigenically homogeneous strains of Bact. Coli neapolitanum from summer diarrhea of infants. Journal of Pathology and Bacteriology, Edenburgh, v. 57, p. 239-247, 1945.

BRUNDER, W.; SCHMIDT, H.; KARCH, H. KatP, a novel catalase-peroxidase encoded by the large plasmid of enterohaemorrhagic Escherichia coli O157:H7. Microbiology, New York, v. 142, n. 11, p. 3305-3315, Nov. 1996.

Esp P, a novel extracellular serine protease of enterohaemorrhagic Escherichia coli $\mathrm{O} 157: \mathrm{H} 7$ cleaves human coagulation factor V. Molecular Microbiology, Salem, v. 24, n. 4, p. 767-778, May 1997.
BÜRK, C.; DIETRICH, R.; AÇAR, G.; MORAVEK, M.; BÜLTE, M.; MÄRTLBAUER, E. Identification and characterization of a new variant of Shiga toxin 1 in Escherichia coli ONT:H19 of bovine origin. Journal of Clinical Microbiology, Washington, v. 41, n. 5, p. 21062112, May 2003.

CALDERWOOD, S. B.; ACHESON, D. W. K.; KEUSCH, G. T.; BARRETT, T. J.; GRIFFIN, P. M.; STOCKBINE, N. A.; SWAMINATHAN, B.; KAPER, J. B.; LEVINE, M. M.; KAPLAN, B. S.; KARCH., H.; O'BRIEN, A. D.; OBRIG, T. G.; TAKEDA, Y.; TARR, P. I.; WACHSMUTH, I. K. Proposed new nomenclature for SLT (VT) family. ASM News, Ann Arbor, v. 62, p. 118-119, Mar. 1996.

CAMPOS, L. C.; FRANZOLIN, M R.; TRABULSI, L. R. Diarrheagenic Escherichia coli among the traditional enteropathogenic E. coli groups - a review. Memórias do. Instituto Oswaldo Cruz, Rio de Janeiro, v. 99, n. 6, p. 545-552, Oct. 2004.

CERQUEIRA, A. M. F.; GUTH, B. E. C.; JOAQUIM, R. M.; ANDRADE, J. R. C. High occurence of Shiga toxinproducing Escherichia coli (STEC) in healthy cattle in Rio de Janeiro State, Brazil. Veterinary Microbiology, Amsterdam, v. 70, n. 1-2, p. 111-121, Oct. 1999.

CORNICK, N. A.; BOOHER, S. L.; MOON, H. W. Intimin facilitates colonization by Escherichia coli $\mathrm{O} 157: \mathrm{H} 7$ in adult ruminants. Infection and. Immunity, Washington, v. 70, n. 5, p. 2704-2707, May 2002.

DEAN-NYSTROM, E. A.; BOSWORTH, B. T.; MOON, H. W.; O'BRIEN, A. D. Escherichia coli O157:H7 requires intimin for enteropathogenicity in calves. Infection and. Immunity, Washington, v. 66, n. 9, p. 4560-4563, Sep. 1998.

DONNENBERG,M.S.;TZIPORI,S.;McKEE,M.L.; O'BRIEN, D. O.; ALROY, J.; KAPER., J. B. The role of eae gene of enterohemorrhagic Escherichia coli in intimate attachment in vitro and in a porcine model. Journal of Clinical Investigation, New York, v. 92, n. 3, p. 1418-1424, Sep. 1993.

DUFFY, G.; GARVEY,P.; MCDOWELL, D. A. Nomenclature of verocytotoxins. In: Verocytotoxigenic Escherichia coli. Trumbull, Ct: Food and Nutrition Press, Inc., p: 447-452. 2001.

ELDER, R. O.; KEEN, J. E.; SIRAGUSE, G. R.; BARKOCYGALLAGHER, G. A.; KOOHMARAIE, M.; LAEGREID, W. W. Correlation of enterohemorrhagic Escherichia coli O157 prevalence in feces, hides, and carcasses of beef cattle during processing. Proceedings of the National Academy of Sciences of the United States of America, Washington, v. 97, n. 7, p. 2999-3003, Mar.2000.

GILES, C.; SANGSTER, G. An outbreak of infantile gastroenteritis in Aberdeen, Journal of Hygiene, Cambridge, v. 46, n. 1, p. 1-9, 1948. 
GIRARD, F.; BATISSON, I.; FRANKEL, G. M.; HAREL, J.; FAIRBROTHER, J. M. Interaction of enteropathogenic and Shiga-toxin producing Escherichia coli and porcine intestinal mucosa: role of intimin and tir in adherence. Infection and Immunity, Washington, v. 73, n. 9, p. 60056016, Sep. 2005.

GRIFFIN, P. M.; TAUXE, R. V. The epidemiology of infections caused by Escherichia coli $\mathrm{O} 157: \mathrm{H7}$, other enterohemorragic E. coli and the associated hemolytic uremic syndrome. Epidemiologic Reviews, Baltimore, v. 13, n. 1, p. 60-98, Jan. 1991.

GUTH, B. E. C.; RAMOS, S. R. T. S.; CERQUEIRA, A. M. F.; ANDRADE, J. R. C.; GOMES, T. A. T. Phenotypic and Genotypic Characteristics of Shiga Toxin producing Escherichia coli Strains Isolated from Children in São Paulo, Brazil. Memórias do Instituto Oswaldo Cruz, Rio de Janeiro, v. 97, n. 8. p. 1085-1089, Dec. 2002.

HABIB, N. F.; JACKSON, M. P. Roles of a ribosomebinding site and mRNA secondary structure in differential expression of Shiga-toxin genes. Journal of Bacteriology, Washington, v. 175, n. 3, p. 597-603, Jan. 1993.

HAYASHI, T.; MAKINO, K.; OHNISHI, M.; KUROKAWA, K.; ISHII, K.; YOKOYAMA, K.; HAN, C.; OHTSUBO, E.; NAKAYAMA, K.; MURATA, T.; TANAKA, M.; TOBE, T.; IIDA, T.; TAKAMI, H.; HONDA, T.; SASAKAWA,C.; OGASAWARA, N.; YASUNAGA, T.; KUHARA, S.; SHIBA, T.; HATTORI, M.; SHINAGAWA, H. Complete genome sequence of enterohemorrhagic Escherichia coli O157:H7 and genomic comparison with a laboratory strain K-12. DNA Research, Amsterdam, v. 8, n. 1, p. 11-22, 2001.

HEAD, S. C.; KARMALI, M., A.; LINGWOOD, C. A. Preparation of VT1 and VT2 hybrid toxins from their purified dissociated subunits. Journal of Biological Chemistry, Bethesda, v. 266, n. 6, p. 3617-3621, Feb. 1991.

IRINO, K.; KATO, M. A. M. F.; VAZ, T. M. I.; RAMOS, I. I.; SOUZA, M.A. C.; CRUZ, A. S.; GOMES, T.A. T.; VIEIRA, M.A. M.; GUTH, B. E. C. Serotypes and virulence markers of Shiga toxin-producing Escherichia coli (STEC) isolated from dairy cattle in São Paulo State, Brazil. Veterinary Microbiology, Amsterdam, v. 105, n. 1, p. 29-36, Jan. 2005.

IRINO, K.; VAZ, T. M. I.; KATO, M. A. M. F.; NAVES, Z. V. F.; LARA, R. R.; MARCO, M. E. C.; ROCHA, M. M. M.; TÂNIAP.; MOREIRA, T. P.; GOMES, T. A. T.; GUTH, B.E. C. O157:H7 Shiga toxin-producing Escherichia coli strains associated with sporadic cases of diarrhea in São Paulo, Brazil. Emerging Infectious Diseases, Atlanta, v. 8, n. 4., p. 446-447, Apr. 2002.

JACKSON, M.P.; NEILL, R. J.; O'BRIEN, A. D.; HOLMES, R. K.; NEWLAND, J. W. Nucleotide sequence analysis and comparison of the structural genes for Shiga-like toxin
I an Shiga-like toxin II encoded by bacteriophages from Escherichia coli 933. FEMS Microbiology Letters,Amsterdam, v. 44, n. 1, p. 109-114, Sep. 1987.

JAEGER, J. L.; ACHESON, D. W. Shiga-toxin producing Escherichia coli. Current Infectious Diseases Reports, London, v. 2, n.1, p.61-67, Fev. 2000.

JENKINS, C.; NEIL T.; PERRY, N.P.; CHEASTY, T.; SHAW, J.D. J.; FRANKEL, G.; DOUGAN, G.; GUNN, G. J.; SMITH, H. R.; PATON, A. W.; PATON, J. C. Distribution of the saa gene in strains of Shiga toxin-producing Escherichia coli of human and bovine origins. Journal of Clinical Microbiology, Washington, v. 41, n. 4, p. 1775-1778, Apr. 2003.

KARMALI, M. A.; STEELE, B. T.; PETRIC, M.; LIM, C. Sporadic cases of haemolytic-uraemic syndrome associated with faecal cytotoxin and cytotoxin-producing Escherichia coli in stools. Lancet, New York, v. 19, n. 1, p. 619-20, Mar. 1983.

KAUFFMANN, F. The serology of the coli group. Journal of Immunology, Baltimore, v. 57, p. 71-100. 1947.

KOCH, C.; HERTWIG, S.; LURZ, R.; APPEL, B.; BEUTIN, L. Isolation of a lysogenic bacteriophage carrying the $s t x 1_{O X 3}$ gene, which is closely associated with Shiga toxinproducing Escherichia coli strains from sheeps and humans. Journal of Clinical Microbiology, Washington, v. 39, n. 11, p. 3992-3998, Nov. 2001.

KONOWALCHUK, J.; SPEIRS, J. L.; STARVIC, S. Vero response to a cytotoxin of Escherichia coli. Infection and Immunity, Washington, v. 18, n. 3, p. 775-779, Dec. 1977.

LAW, D.; KELLY, J. Use of heme and hemoglobin by Escherichia coli $\mathrm{O} 157$ and other Shiga-like-toxinproducing E. coli serogroups. Infection and Immunity, Washington, v. 63, n. 2, p. 700-702, Feb. 1995.

LEOMIL, L.; AIDAR-UGRINOVICH, L.; GUTH, E. E. C.; IRINO, K.; VETTORATO, M. P.; ONUMA, D. L.; CASTRO, A. F. P. Frequency of Shiga toxin-producing Escherichia coli (STEC) isolates among diarrheic and non-diarrheic calves in Brazil. Veterinary Microbiology, Amsterdan, v. 97, n. 1-2, p. 103-109, Dec. 2003.

LEVINE, M. M;, XU, J.; KAPER, J. B.; LIOR, H.; PRADO, V.; TALL, B.; NATARO, J.; KARCH, H.; WASCHSMUTH, K. A DNA probe to identify enterohemorrhagic Escherichia coli of O157:H7 and other serotypes that cause hemorrhagic colitis and hemolytic uremic syndrome. Journal of Infectious Diseases, v. 156, n. 1, p. 175-182, Jul. 1987.

LUCK, S. N.; BENNETT-WOOD,V.; POON, R.; ROBINSBROWNE, R. M.; HARTLAND, E. L. Invasion of Epithelial Cells by Locus of Enterocyte Effacement-Negative Enterohemorrhagic Escherichia coli. Infection and Immunity, Washington, v. 73, n. 5, p. 3063-3071, May 2005. 
MAINIL, J. G.; DAUBE, G. Verotoxigenic Escherichia coli from animal, humans and foods: who's who? Journal of Applied Microbiology, Oxford, v. 99, n. 6, p. 1332-1344, Dec. 2005.

McKEE, M. L.; MELTON-CELSA, A. R.; MOXLEY, R. A.; FRANCIS, D. H.; O'BRIEN, A. D. Enterohemorrhagic Escherichia coli $\mathrm{O} 157: \mathrm{H} 7$ requires intimin to colonize the gnotobiotic pig intestine and to adhere to HEp-2 cells. Infection and Immunity, Washington, v. 63, n. 9. p. 37393744, Sep. 1995.

MEAD, P. S.; SLUTSKER, L.; DIETZ, V.; MCCAIG, L. F.; BRESEE, J. S.; SHAPIRO, C.; GRIFFIN, P. M.; TAUXE, R. V. Food-related illness and death in the United States. Emerging Infectious Diseases, Atlanta, v. 5, n. 5, p. 607625, Sep./Oct. 1999.

MELTON-CELSEA, A. R.; O'BRIEN, A. D. Structure, biology and relative toxicity of Shiga toxin family members for cells and animals. In: KAPER, J. B.; O'BRIEN, A. D. Escherichia coli O157:H7 and other Shiga-toxin producing E. coli strains. Washington, DC: ASM Press, 1998. p. 121-128.

MOREIRA, C. N.; PEREIRA, M. A.; BROD, C. S.; RODRIGUES, D. P.; CARVALHAL, J. B.; ALEIXO, J. A. G. Shiga-toxin-producing Escherichia coli (STEC) from healthy dairy cattle in southern Brazil. Veterinary Microbiology, Amsterdam, v. 93, n. 3, p. 179-183, May 2003.

NAKAJIMA, H.; KIYOKAWA, N.; KATAGIRI, Y. U.; TAGUCHI, T.; SUZUKI, T.; SEKINO, T.; MIMORI, K.; EBATA, T.; SAITO, M.; NAKAO, H.; TAKEDA, T.; FUJIMOTO, J. Kinetic Analysis of Binding between Shiga Toxin and Receptor Glycolipid Gb3Cer by Surface Plasmon Resonance. Journal of Biological Chemistry, Bethesda, v. 276, n. 46, p. 42915-42922, Nov. 2001.

NATARO, J. P.; KAPER, B. Diarrheagenic Escherichia coli. Clinical Microbiology Reviews, Washington, v. 11, n. 1, p. 142-201, Jan. 1998.

NICHOLLS, L.; GRANT, T. H.; ROBINS-BROWNE, R. M. Identification of a novel genetic locus that is required for in vitro adhesion of a clinical isolate of enterohaemorrhagic Escherichia coli to epithelial cells. Molecular Microbiology, Salem, v. 35, n. 2, p. 275-288, Jan. 2000.

O'BRIEN, A. D.; LA VECK, G. D.; THOMPSON, M. R.; FORMAL, S. B. Production of Shigella dysenteriae type 1-like citotoxin by Escherichia coli. Journal of Infectious Diseases, Chicago, v. 146, n. 6, p. 763-769. 1982.

O'BRIEN, A. D.; LA VECK, G. D. Purification and characterization of a Shigella dysenteriae 1-like toxin produced by Escherichia coli. Infection and Immunity, Washington, v. 40, n. 1, p. 675-683, May 1983.
O'BRIEN, A. D.; LIVELY, T. A.; CHEN, M.E.; ROTHMAN, S. W.; FORMAL, S. B. Escherichia coli O157:H7 strains associated with haemorrhagic colitis in the United States produce a Shigella dysenteriae 1 (Shiga) like cytotoxin. The Lancet, New York, v. 26, n. 1, p. 702, Mar.1983.

O'BRIEN, A. D.; HOLMES, R. K. Protein toxins of Escherichia coli and Salmonella. In: NEIDHARDT, F.C.; CURTISS III, R.; INGRAHAM, J. L.; LIN, E. C. C.; LOW, K. B.; MAGASANIK, B.; REZNIKOFF, B. S.; RILEY, M.; SCHAECHTER, M.; UMBARGER, H.E. (Ed.). Escherichia coli and Salmonella: cellular and molecular biology. Washington, DC: ASM Press, 1986. p.2788-2802.

OSEK J.; WEINER, M.; HARTLAND, E.L. Prevalence of the lpfO113 gene cluster among Escherichia coli $\mathrm{O} 157$ isolates from different sources. Veterinary Microbiology, Amsterdam, v. 96, n. 3, p. 259-266, Oct. 2003.

PADOLA, N. L.; SANZ, M. E.; BLANCO, J. E.; BLANCO, M.; BLANCO, J.; ETCHEVERRIA, A. I.; ARROYO, G. H.; USERA, M. A.; PARMA, A. E. Serotypes and virulence genes of bovine Shigatoxigenic Escherichia coli (STEC) isolated from a feedlot in Argentina. Veterinary Microbiology, Amsterdam, v. 100, n. 1, p. 3-9, May 2004.

PATON, J. C.; PATON, A. W. Patogenesis and diagnosis of Siga toxin-producing Escherichia coli infections. Clinical Microbiology Reviews, Washington, v. 11, n. 3, p. 450-479, Jul. 1998.

PATON, A. W.; SRIMANOTE, P.; WOODROW, M. C.; PATON, J. C. Characterization of Saa, a novel autoagglutinating adhesin produced by locus of enterocyte effacement-negative Shiga-toxigenic Escherichia coli strains that are virulent for humans. Infection and Immunity, Washington, v. 69, n. 11, p. 6999-7009, Nov. 2001.

PERNA, N. T.; PLUNKETT, G.; BURLAND, V.; MAU, B.; GLASNER, J. D.; ROSE, D. J.; MAYHEW, G., F.; EVANS, P. S.; GREGOR, J.; KIRKPATRICK. H. A.; PÓSFAI, G.; HACKETT, J.; KLINK, S.; BOUTIN, A.; SHAO, Y.; MILLER, L.; GROTBECK, E. J.; DAVIS, W.; LIMK, A.; DIMALANTAK, E. T.; POTAMOUSIS, K. D.; APODACA, J.; ANANTHARAMAN, T. S.; LIN, J.; YEN, G.; SCHWARTZ, D. C.; WELCHI, R. A.; BLATTNER, F. R. Genome sequence of enterohaemorrhagic Escherichia coli O157:H7. Nature, London, v. 409, p. 529-533, Jan. 2001.

RILEY, L. W.; REMIS, R. S.; HELGERSON, S. D.; MCGEE, H. B.; WELLS, J. G.; DAVIS, B. R.; HEBERT., R. J.; OLCOTT, E. S.; JOHNSON, L. M.; HARGRETT, N. T.; BLAKE. P. A.; COHEN, M. L. Hemorrhagic colitis associated with a rare Escherichia coli serotype. New England Journal of Medicine, Walthan, v. 308, n. 12, p. 681-685, Mar. 1983.

RILEY, L. W. The epidemiological, clinical and microbiologic features of haemorragic colitis. Annual Review of Microbiology, Palo Alto, v. 41, p. 383-407, Oct. 1987. 
ROBINS-BROWNE, R. M. Traditional enteropathogenic Escherichia coli of infantile diarrhea. Reviews of Infectious Diseases, Chicago, v. 9, n. 1, p. 28-53, 1987.

SALVADORI, M. R.; VALADARES, G. F.; LEITE, D. S.; BLANCO, J.; YANO, T. Virulence factors of Escherichia coli isolated from calves with diarrhea in Brazil. Brazilian Journal of Microbiology, São Paulo, v. 34, n. 3, p. 230235, Sep. 2003.

SANZ, M. E.; VIÑAS, M. R.; PARMA, A. E. Prevalence of bovine verotoxin-producing Escherichia coli in Argentina. European Journal of Epidemiology, Dordrecht, v. 14, n. 4, p. 399-403, Jun. 1998.

SCHMIDT, H.; KARCH, H.; BEUTIN, L. The large-sized plasmids of entrohemorragic Escherichia coli O157 strains encode hemolysins which are presumably members of the E. coli a-hemolysin family. FEMS Microbiology Letters, Amsterdam,, v. 117, n. 2.p. 189-196, Apr. 1994.

SMITH, A.G.; HILLIER, J.; MUDD, S. Internal structure and nuclei in cells of Escherichia coli as shown by improved electron microscopic techniques. Journal of Bacteriology, Washington, v. 57, n. 3, Mar. 1949.

STEVENS, M.P.; VAN DIEMEN, P.M.; FRANKEL, G.; PHILLIPS, A.D.; WALLIS, T.S.. Efa1 influences colonization of the bovine intestine by Shiga toxinproducing Escherichia coli serotypes O5 and O111. Infection and Immunity, Washington, v. 70, n. 9, p. 51585166, Sep. 2002.

SWINBANKS, D. Japan shuns radishes after 'possible link' to E. coli. Nature, London, v. 382, p. 567, Aug. 1996.

SZALO, I. M.; GOFFAUX, F.; PIRSON, V.; PIÈRARD, D.; BALL, H.; MAINIL, J. Presence of bovine enteropathogenic (EPEC) and enterohaemorrhagic (EHEC) Escherichia coli of genes encoding for putative adhesins of human EHEC strains. Research in Microbiology, Paris, v. 153, n. 10, p. 653-658, Dec. 2002.

TARR, P. I.; BILGE, S. S.; VARY, JR., J. C.; JELACIC, S.; HABEEB, R. L.; WARD, T. R.; BAYLOR, M. R.; BESSER, T. E. Iha: a novel Escherichia coli O157:H7 adherenceconferring molecule encoded on a recently acquired chromosomal island of conserved structure. Infection and Immunity, Washington, v. 68, n. 3, p. 1400-1407, Mar. 2000.
THORPE, C. M.; RITCHIE, J. M.; ACHESON, D. W. K. Enterohemorrhagic and other Shiga toxin producing Escherichia coli. In: DONNENBERG, M. S. (Ed.). Escherichia coli: Virulence mechanisms of a versatile pathogen. San Diego, CA: Academic Press, 2002. p.119-154.

TORRES, A. G.; GIRON, J. A.; PERNA, N. T.; BURLAND, V.; BLATTNER, F. R.; AVELINO-FLORES, F.; KAPER, J. B.

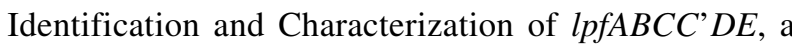
Fimbrial Operon of Enterohemorrhagic Escherichia coli O157:H7. Infection and Immunity, Washington, v. 70, n. 10, p. 5416-5427, Oct. 2002a.

TORRES,A. G; PERNA, N. T.; BURLAND, V.; RUKNUDIN, A.; BLATTNER, F. R.; KAPER, J. B. Characterization of Cah, a calcium-binding and heat-extractable autotransporter protein of enterohaemorrhagic Escherichia coli.. Molecular Microbiology, Salem, v. 45, n. 4, p. 951, Aug. 2002b.

TORRES, A. G.; KAPER, J. B. Multiple elements controlling adherence of enterohemorrhagic Escherichia coli O157:H7 to HeLa Cells. Infection and Immunity, Washington, v. 71, n. 9, p. 4985-4995, Sep. 2003.

VAN DIEMEN, P. M.; DZIVA, F.; STEVENS, M. P.; WALLIS, T. S. Identification of enterohemorrhagic Escherichia coli $\mathrm{O} 26: \mathrm{H}^{-}$genes required for intestinal colonization in calves. Infection and Immunity, Washington, v. 73, n. 3, p. 1735-1743, Mar. 2005.

VAZ, T. M. I.; IRINO, K.; KATO, M. A. M. F.; DIAS, Â. M. G.; GOMES, T. A. T.; MEDEIROS, M. I. C.; ROCHA, M. M. M.; GUTH, B. E. C. Virulence Properties and Characteristics of Shiga Toxin-Producing Escherichia coli in São Paulo, Brazil, from 1976 through 1999. Journal of Clinical Microbiology, Washington, v. 42, n. 2, p. 903905, Feb. 2004.

VERWEYEN, H. M.; KARCH, H.; BRANDIS, M.; ZIMMERHACKL, L. B. Enterohemorrhagic Escherichia coli infections: following transmission routes. Pediatric Nephrology, Berlim, v. 14, n. 1, p. 73-83, Oct. 2000.

ZHANG, W.; BIELASZEWSKA, M., KUCZIUS, P.; $\mathrm{KARCH}, \mathrm{H}$. Identification, characterization and distribution of a Shiga toxin 1 gene variant (stx1c) in Escherichia coli strains isolated from humans. Journal of Clinical Microbiology, Washington, v. 40, n. 4, p. 14411446, Apr. 2002. 
